\title{
RELCASI
}

\section{Estudio Exploratorio de los Departamentos de Tecnologías de Información}

\author{
Juan M. Gómez Reynoso \\ Universidad Autónoma de Aguascalientes, jmgr@correo.uaa.mx \\ Carlos A. Torres Quiñones \\ Sofftek, carlostq@hotmail.com \\ Javier S. Cortes López \\ Gobierno del Estado de Aguascalientes,, scortes@gruposinco.com.mx
}

Follow this and additional works at: https://aisel.aisnet.org/relcasi

\section{Recommended Citation}

Gómez Reynoso, Juan M.; Torres Quiñones, Carlos A.; and Cortes López, Javier S. (2008) "Estudio Exploratorio de los Departamentos de Tecnologías de Información," RELCASI: Vol. 1 : Iss. 1 , Paper 2. DOI: 10.17705/1 relc.00003

Available at: https://aisel.aisnet.org/relcasi/vol1/iss1/3

This material is brought to you by the AIS Journals at AIS Electronic Library (AISeL). It has been accepted for inclusion in RELCASI by an authorized administrator of AIS Electronic Library (AISeL). For more information, please contact elibrary@aisnet.org. 


\section{$\begin{array}{lllllllll}\text { R I L C A S I } & 01 & 01 & 2008\end{array}$}

\section{REVISTA LAINOAMERICANA}

Y DEL CARIBE DE LA

ASOCIACIÓN DE SISIEMAS

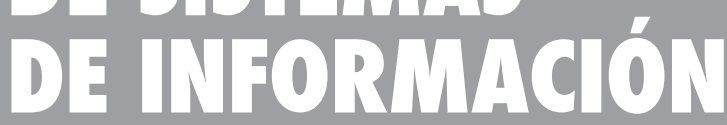

11 - Utilización de la Metodología

Mixta de Investigación en SI:

El caso del estudio de la Cultura

Ocupacional de Sistemas Indira R. Guzmán

31 - Estudio Exploratorio de los Departamentos de Tecnologías de Información Juan Manuel Gómez Reynoso, Carlos Alejandro Torres Quiñones y Javier Santiago Cortes López

53 - Factores Decisivos para el Éxito de la Capacitación Corporativa con Base en la Web en Brasil: Una Investigación Exploratoria Multicaso Luiz Antonio Joia y Mário Figueiredo Costa

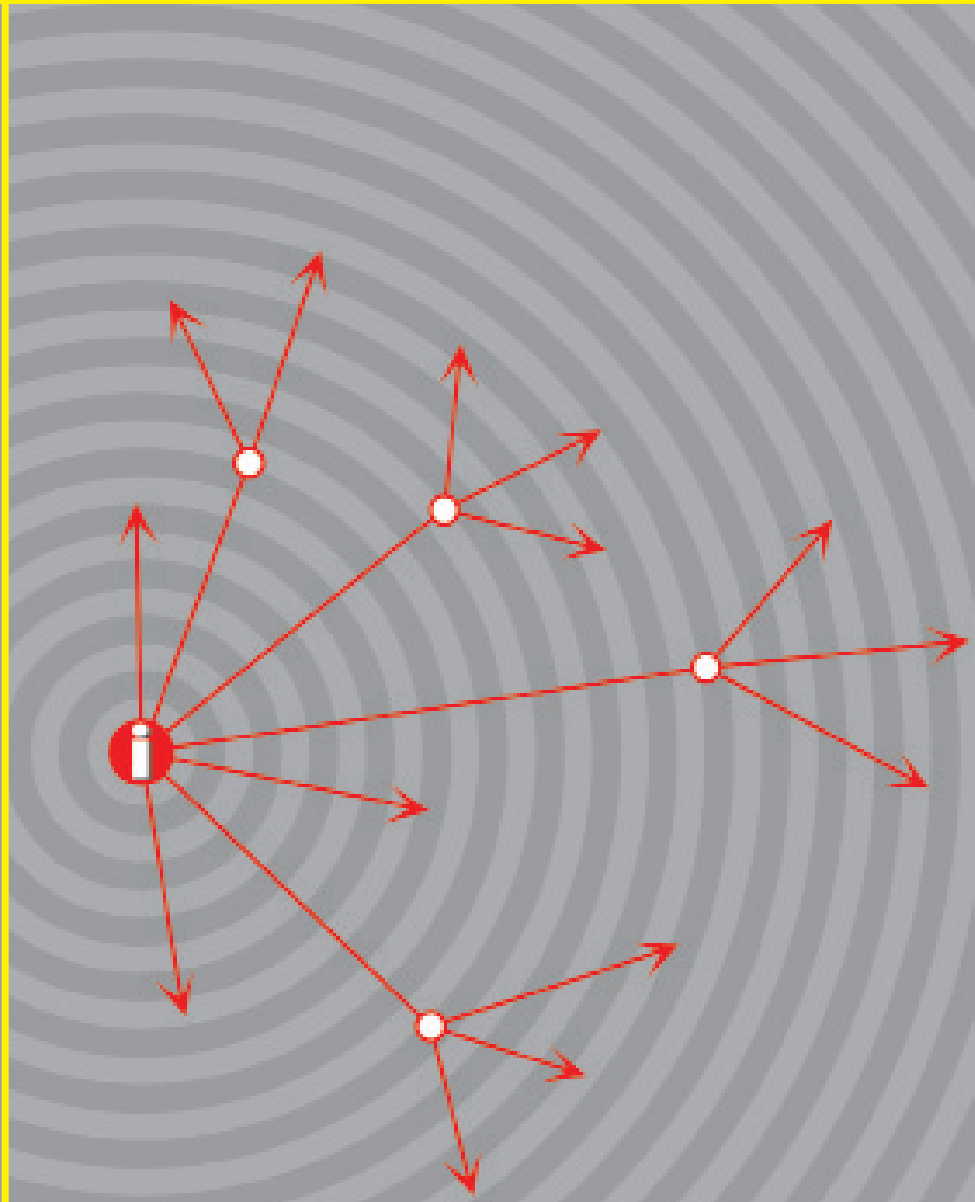




\section{$\begin{array}{lllllllllll}\mathbf{R} & \mathbf{E} & \mathbf{L} & \mathbf{C} & \mathbf{A} & \mathbf{S} & \mathbf{I} & & 01 & 01 & 2008\end{array}$}

\section{REVISTA LAIINOAMERICANA}

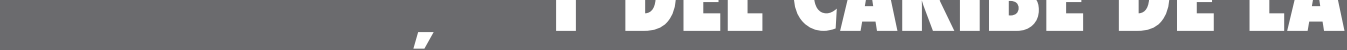
ASOCIACIÓN DE SISTIMAS DE INFORMAción

Editor:

Carlos Ferran 
Carlos Fe

Editor:

Penn State Great Valley Pennsylvania State University 30 E Swedesford Road

Malvern, PA 19355

U.S.A.

cferran@psu.edu

Comité Editorial:

Carlos Dorantes

Tecnológico de Monterrey, México

cdorante@itesm.mx

Carlos Ferran

Pennsylvania State University,

USA

cferran@psu.edu

Martha Garcia-Murillo

Syracuse University, USA

mgarciam@syr.edu

David Montesinos

INCAE, Costa Rica

Montesin@mail.incae.ac.cr

Carlos J. Navarrete California State Polytechnic University, USA

cjnavarrete@csupomona.edu

James B. Pick

University of Redlands, USA

James_Pick@redlands.edu

Guillermo Rodríguez-Abitia

Tecnológico de Monterrey,

Campus Estado de México

grdrz@itesm.mx

Martin Santana

ESAN, Peru

MSantana@esan.edu.pe

Volumen 1 Número 1, 2008

Portada: Maria Elena Repiso

(c) 2008 RELCASI

ISSN 1937-8823

(en línea) ISSN 1937-8831

www.relcasi.org

\section{Revista Latinoamericana} y del Caribe de la Asociación de Sistemas de Información RELCASI

\section{TABLA DE CONTENIDO}

Volumen 1 Número 1, 2008

INTRODUCCIÓN EDITORIAL

UTILIZACIÓN DE LA METODOLOGÍA MIXTA DE INVESTIGACIÓN EN SI: EL CASO DEL ESTUDIO DE LA CULTURA OCUPACIONAL DE SISTEMAS

Indira R. Guzmán

ESTUDIO EXPLORATORIO DE LOS DEPARTAMENTOS DE TECNOLOGÍAS DE INFORMACIÓN

Juan Manuel Gómez Reynoso,

Carlos Alejandro Torres Quiñones y

Javier Santiago Cortes López

FACTORES DECISIVOS PARA EL ÉXITO DE LA CAPACITACIÓN CORPORATIVA CON BASE EN LA WEB EN BRASIL: UNA INVESTIGACIÓN EXPLORATORIA MULTICASO

Luiz Antonio Joia y

Mário Figueiredo Costa

POLÍTICA EDITORIAL 
Editor-in-Chief: Carlos Ferran Penn State Great Valley Pennsylvania State University 30 E Swedesford Road Malvern, PA 19355 U.S.A. cferran@psu.edu Editorial Board:

Carlos Dorantes Tecnológico de Monterrey, México cdorante@itesm.mx

Carlos Ferran Pennsylvania State University, USA cferran@psu.edu Martha Garcia-Murillo Syracuse University, USA mgarciam@syr.edu

David Montesinos INCAE, Costa Rica Montesin@mail.incae.ac.cr

Carlos J. Navarrete California State Polytechnic University, USA cjnavarrete@csupomona.edu

James B. Pick University of Redlands, USA James_Pick@redlands.edu

Guillermo Rodríguez-Abitia Tecnológico de Monterrey, Campus Estado de México grdrz@itesm.mx

Martin Santana ESAN, Peru MSantana@esan.edu.pe

Volume 1 Number 1, 2008 (C) 2008 RELCASI Cover: Maria Elena Repiso ISSN 1937-8823 (on-line) ISSN 1937-8831 www.relcasi.org

\section{Revista Latinoamericana y del Caribe de la Asociación de Sistemas de Información RELCASI}

TABLE OF CONTENT

Volume 1 Number 1, 2008

EDITORIAL INTRODUCTION

USINGMIXEDMETHODOLOGYININFORMATIONSYSTEMS: THE CASE OF THE STUDY OF THE IT OCCUPATIONAL CULTURE 11 Indira R. Guzmán

ANEXPLORATORYSTUDYOFTHEINFORMATIONTECHNOLOGY DEPARTMENTS

Juan Manuel Gómez Reynoso,

Carlos Alejandro Torres Quiñones y

Javier Santiago Cortes López

CRITICAL SUCCESS FACTORS IN WEB-BASED CORPORATE TRAINING IN BRAZIL: AN EXPLORATORY MULTI-CASE INVESTIGATION

Luiz Antonio Joia y

Mário Figueiredo Costa

EDITORIAL POLICY

CALL FOR ARTICLES 


\title{
ESTUDIO EXPLORATORIO DE LOS DEPARTAMENTOS DE TECNOLOGÍAS DE INFORMACIÓN \\ AN EXPLORATORY STUDY OF THE INFORMATION TECHNOLOGY DEPARTMENTS
}

\author{
Juan Manuel Gómez Reynoso \\ Universidad Autónoma de Aguascalientes, jmgr@correo.uaa.mx \\ Carlos Alejandro Torres Quiñones \\ Sofftek, carlostq@hotmail.com \\ Javier Santiago Cortes López \\ Gobierno del Estado de Aguascalientes, scortes@gruposinco.com.mx
}

\section{RESUMEN}

El presente estudio trata acerca de los aspectos relativos a la administración de los departamentos de TI en una organización, generando una herramienta que permitirá explorar su grado de madurez. El estudio está enfocado en los procesos de las áreas de Administración y Evaluación de TI, tomando como caso particular el estado de Aguascalientes, México. La evaluación se realizó mediante un análisis de factores, el cual arrojó un total de 12 factores; 6 utilizables (Satisfacción del Usuario, Portafolio de Aplicaciones, Capital Humano, Estructura Organizacional, Modelo de Calidad, Recursos Financieros) y 3 que debieron ser eliminados. Además, se efectuó un análisis de confiabilidad usando el Alfa de Cronbach.

Palabras claves: CMMI, IT Service CMM, madurez, Benchmarking, Tecnologías de Información.

\section{ABSTRACT}

The present study explores the management aspects related to the organizations' IT departments developing a tool that allows its maturity level measurement. Our study focuses on the processes related to the Management and Evaluation of IT. It was conducted in Aguascalientes, Mexico as a case study. The evaluation was made using a factor analysis approach, which produced a set of 12 factors; 6 usable (User Satisfaction, Applications Portfolio, Human Capital, Organization Structure, Quality Model, Financial Resources) and 3 that have to be eliminated. An additional reliability analysis using Cronbach Alpha was also performed.

Keywords: CMMI, IT Service CMM, maturity level, Benchmarking, Information Technology. 


\section{INTRODUCCIÓN}

El grado de madurez de las Tecnologías de Información (TI) permite detectar, en forma indirecta, su nivel de aceptación y utilización. La administración debe atender este problema, y en su caso, resolverlo. Este asunto tiene un carácter general que impacta en toda la organización, especialmente a los centros responsables de la administración de los sistemas de información.

El entender los factores que influyen en el comportamiento de un departamento de TI en las organizaciones es importante, ya que la existencia de éstos afecta prácticamente a todas las áreas. Por eso es importante detectar los principales problemas en los departamentos de TI (Crook, 1997; Ginzberg \& Baroudi, 1992).

Para la presente investigación se encuestaron un total de 126 personas, las cuales trabajan en los departamentos de TI de organizaciones en 7 industrias diferentes. Los resultados identifican un total de 9 factores que fueron considerados como críticos. Cabe mencionar que 3 de ellos debieron ser eliminados por estar conformados por una sola variable. Además, se realizó una medición del Alfa de Cronbach con el fin de medir la confiabilidad de los 6 factores identificados como clave. Como valor de corte se utilizo .7 de confiabilidad. Todos los factores identificados tuvieron ese valor o más como confiabilidad.

El artículo se forma de la siguiente manera. Primero, se muestra la revisión bibliográfica realizada; enseguida, se explica la forma en que se conformó el modelo, el cual genera el instrumento aplicado; posteriormente, se describe la metodología de investigación utilizada; a continuación, se discuten los resultados obtenidos; terminando con las recomendaciones, limitaciones, así como los trabajos futuros propuestos.

\section{REVISIÓN BIBLIOGRÁFICA}

Investigaciones pasadas han tratado de crear modelos que permitan evaluar las organizaciones. Esta evaluación permite detectar problemas con el fin de mejorar la calidad de los procesos, productos y servicios. Por ejemplo, Nolan (1973) propuso una teoría para la administración del recurso, la cual se basa en que las actividades de planeación, organización y control asociadas a la administración del recurso computacional cambian y evolucionan en un período de tiempo. Además, Nolan describe seis etapas para el crecimiento en el procesamiento de datos en las organizaciones y explica que dichas etapas capturan las tendencias centrales en las principales tareas de administración de planeación, organización y control del recurso computacional. Las actividades principales en la administración de un área de informática son identificables en etapas que corresponden a períodos de estabilidad en el crecimiento del uso de cómputo en la organización, es decir, es posible 
detectar en qué fase del modelo se encuentra un área de informática a partir de una serie de factores que nos permiten determinar su nivel de madurez. La idea principal detrás de los modelos de crecimiento es que las cosas cambian con respecto al tiempo y las organizaciones van madurando igualmente (Janssen, Gortmaker, \& Wagenaar, 2006). Cabe mencionar que el uso de TI y sistemas de información (SI) en las organizaciones ha crecido y cambiado permanentemente (Delmonte, 2003). Leem y Kim (2004) mencionan que, típicamente, los usuarios de SI esperan utilizar varias aplicaciones en las organizaciones. Más aún, la mayoría de las organizaciones intentan renovar los procesos de negocios y sus estrategias a través de los SI. En consecuencia, la aplicación de metodologías y/o técnicas de mejora de procesos y productos/servicios permiten a los departamentos de TI de las organizaciones el coadyuvar en la planeación estratégica.

Los modelos de evaluación de los procesos de software miden el nivel de capacidad que se tiene, con la meta de mejorar los procesos de desarrollo (Leem \& Yoon, 2004), algunos de ellos son: Capability and Maturity Models (CMM), Software Process Improvement and Capability Determination (SPICE) y Personal Software Process (PSP). El Capability Maturity Model Integration (CMMI) es una metodología para mejorar los procesos de desarrollo de software con la meta de entregar software de alta calidad a tiempo y dentro de presupuesto (Agrawal \& Chari, 2007). CMMI está compuesto por cinco etapas relacionadas al nivel de madurez (Agrawal \& Chari, 2007; Leem \& Kim, 2004; Leem \& Yoon, 2004). Además, garantiza la entrega de un producto a través de asegurar todas las áreas del proceso (C.M.U., 2000). Farris et al. (2007) argumentan que la evaluación de la capacidad de cualquier proceso usando CMMI incluye la evaluación tanto de la calidad de salida del proceso como de la calidad del proceso usado en la aplicación de las prácticas específicas. Por ejemplo, CMMI permitió una reducción del $60 \%$ en el trabajo a Boeing, mientras que Lockheed Martin incrementó un 30\% la productividad del software (Agrawal \& Chari, 2007). Entonces, CMMI permite mejorar los procesos individuales de producción de software (Farris et al., 2007), haciendo más eficientes a las organizaciones que lo adoptan.

IT Service CMM es el producto de dos proyectos auspiciados que trataban de desarrollar un método para especificar y controlar servicios de TI (van Vlient, 1999) y está basado en CMM; éste está orientado tanto al aseguramiento de la capacidad de los servicios de TI, así como a las directrices necesarias para su mejora (F. Niessink \& Van Vlient, 1999). IT Service CMM es un modelo de madurez de los procesos que entrega servicios de TI a los usuarios. Además, cubre no sólo los productos de TI sino también servicios tales como mantenimiento, reparación, administración de la red y educación, entre otros (Leem \& Yoon, 2004). Es importante mencionar que IT Service CMM es un esfuerzo que aún no 
termina y que está en desarrollo y definición de niveles. La estructura tanto de CMMI como de IT Service CMM es muy similar. Cada proceso clave está estructurado utilizando prácticas comunes que al ejecutarse en conjunto garantizan la mejora en los procesos del desarrollo del proyecto (C.M.U., 2000).

La madurez es un estado de equilibrio, en el cual el recurso computacional está bajo control por medio de acciones de administración apropiadas y persistentes hasta que los nuevos cambios tecnológicos perturben el equilibrio (King \& Kraemer, 1984). Para este estudio, se tomó como madurez la definición dada por Delmonte (2003), como la planeación formal y el control de los sistemas, así como los sistemas de cómputo que se integran en los procesos de planeación estratégica de la organización. El presente estudio tiene como objetivo el diseño de un instrumento conceptual que pueda servir para obtener un acercamiento real al nivel de madurez de los departamentos de TI en las organizaciones. Para poder determinar la validez del instrumento se llevó a cabo su aplicación y evaluación en las empresas del área metropolitana de la ciudad de Aguascalientes, México; de esta forma servirá como estudio exploratorio en este campo.

Drucker (1999) menciona que sólo se puede administrar lo que se puede medir. Es por ello la importancia de las mediciones en el conocimiento de algún nivel de madurez en los departamentos de TI. La mayoría de los departamentos de TI utilizan mediciones para justificar presupuestos, pero sólo una pequeña parte ha tratado de explotarlos para la búsqueda de algún mejoramiento sustancial (Due, 1996). Uno de los primeros pasos para introducir de manera apropiada mediciones de la Productividad fue la aplicación de un análisis de puntos de función desarrollado por Albrecht en IBM a mediados de los 70s. El análisis de puntos de función permite determinar el esfuerzo requerido para el desarrollo de sistemas que efectúa el personal de los departamentos de TI (Pressman, 2005; Sommerville, 2006). Los puntos de función miden el tamaño del software. Éstos miden la funcionalidad mediante la medición objetiva de los requerimientos funcionales del software. Además, cuantifican y documentan las suposiciones que se hacen en la estimación del desarrollo de software. Más aún, éstos son independientes de la tecnología y de la metodología de diseño utilizados. Sin embargo, la productividad de un departamento de TI sólo puede ser efectivamente medida cuando se tiene una perspectiva de toda la productividad de la organización. Cabe mencionar que el fenómeno acerca del nivel de productividad ha sido ampliamente estudiado (Brynjolfsson \& Hitt, 1996; Hitt \& Brynjolfsson, 1996) y se afirma que lo que se obtiene del uso de TI no está en relación con lo que se invierte en TI. No obstante, todas las organizaciones definen de una forma u otra la productividad para sus empleados, por ejemplo, número de problemas resueltos, tiempo de atención al cliente, por mencionar algunos. Es por 
ello la importancia de las métricas en el conocimiento de algún nivel de madurez en los departamentos de TI (Due, 1996).

\section{MODELO PROPUESTO}

El presente estudio intenta describir el nivel de madurez de los departamentos de TI en las organizaciones. Con el fin de realizar el estudio exploratorio, se decidió integrar cuatro teorías base, las cuales tratan de una forma u otra la madurez de los departamentos de TI. Los diferentes modelos/teorías consultados: teoría de etapas de Nolan (1973), CMMI (Agrawal \& Chari, 2007; C.M.U., 2000; Leem \& Kim, 2004; Leem \& Yoon, 2004), IT Service CMM (F. Niessink \& van Vliet, 1999; van Vlient, 1999), Benchmarking y Métricas de TI (Gordon, 1994) exponen de una forma u otra el comportamiento de un departamento de TI en una organización. La Tabla 1 muestra los puntos comunes encontrados en las diferentes teorías, la cual fue construida a partir del análisis de aquellas que estudian la madurez de los departamentos de TI. Asimismo, los factores considerados se describen a continuación.

Satisfacción del Usuario. De acuerdo a Nolan (1973), las aplicaciones de computadora tienen un impacto en la satisfacción de los usuarios. Asimismo, la satisfacción del usuario debe ser parte de los componentes de la calidad y el desempeño de los procesos (C.M.U., 2000). Niessink y van Vlient (2000) mencionan que las acciones de los empleados impactan el servicio y la satisfacción de los usuarios. Además, Gordon (1994) menciona que la satisfacción el usuario es un elemento de comparación (benchmark) muy importante.

Personal. Existe un nivel alto de competencia entre las organizaciones por contratar personal de sistemas capacitado, Nolan (1973). Los requerimientos de contratación de personal dependen del conocimiento y habilidades disponibles para dar soporte a la ejecución del proyecto (C.M.U., 2000). Es importante agregar que los empleados son parte primordial del esfuerzo de benchmarking que se realiza (Gordon, 1994).

Recursos Financieros. Nolan describe que la curva del presupuesto servirá como un sustituto útil para representar el fenómeno del crecimiento. Asimismo, Gordon (1994) menciona que es muy importante que los proyectos se finalicen dentro de presupuesto y a tiempo. Adicionalmente, investigaciones pasadas (F. Niessink \& van Vliet, 1999; van Vlient, 1999) explican que es importante presupuestar el soporte que se da a los grupos involucrados en la prestación de servicios. Más aún, el presupuesto es parte primordial de todo proyecto de sistemas (C.M.U., 2000).

Tecnología. Nolan (1973) sugiere que la planeación, organización y actividades de control asociadas al manejo del recurso computacional cambian con respecto al tiempo, evolucionando en patrones algo relacionados a las cuatro etapas, así como la obvia complejidad de la gerencia en general, y la TI en específico, contribuyen al escepticismo 
generado alrededor de ésta. La tecnología es un aspecto muy importante que se discute para CMMI (C.M.U., 2000), así como un proceso clave de IT Service CMM (F. Niessink \& Van Vlient, 1999; F. Niessink \& van Vlient, 2000). Adicionalmente, Gordon (1994) describe que la tecnología tiene un impacto muy fuerte en las organizaciones ya que la inversión en ella impacta el retorno de la inversión y el desempeño global.

Alineamiento hacia el negocio. La gerencia ha buscado activamente la clave para explotar eficazmente el recurso computacional con el fin de aprovechar las oportunidades de negocios (Nolan, 1973).

Portafolio de Aplicaciones. Nolan (1973) describe que existe un conjunto de variables situacionales, junto a un conjunto de tareas y sus atributos dan como resultado el perfil de las aplicaciones de TI en las organizaciones. Las aplicaciones desarrolladas de TI han impactado considerablemente el funcionamiento de las organizaciones, aunque en muchas ocasiones el costo sea más elevado (Nolan, 1973) que lo presupuestado. Es muy importante tener un conocimiento profundo de la organización, del ámbito del negocio y de las circunstancias implicadas. Las organizaciones deben interpretar las prácticas del negocio de forma tal que trabajen para su organización. El término "activos del proceso" se utiliza para indicar los artefactos desarrollados o adquiridos para lograr los objetivos de la organización, y éstos representan las inversiones de la organización que se espera que proporcionen valor al negocio actual y futuro (C.M.U., 2000). Una de las metas de hacer benchmarking consiste en mejorar las aplicaciones existentes en la organización (Gordon, 1994), apalancándolas con TI con el fin de incrementar el desempeño del negocio. Los procesos de desarrollo de sistemas que usan las organizaciones dependen de múltiples factores, incluyendo el tamaño, la estructura y el dominio particular de la organización (C.M.U., 2000), sin importar que los sistemas a desarrollar/mantener no sean únicos. Gordon (1994) menciona que la mayoría de las organizaciones desarrolla y compra TI realizando antes rigurosos análisis de retorno de la inversión. En consecuencia, las aplicaciones de TI que una organización compra o desarrolla dependen de sus necesidades, pero siempre teniendo en mente el retorno de la inversión.

La Organización. La gerencia reconoce el hecho de que la TI impacta a la organización; entonces, es deseable intentar que la TI sea asimilada por los empleados con el menor problema posible (Nolan, 1973). A muchas organizaciones les gustaría enfocar sus esfuerzos de mejora (C.M.U., 2000). Sin embargo, las diferencias entre los modelos que aplican, han limitado la capacidad de éstas organizaciones en realizar sus mejoras con éxito. Además, la aplicación de modelos múltiples que están integrados dentro y a través de la organización llega a ser más costosa en términos de entrenamiento, evaluación y actividades de la mejora. Las organizaciones adquieren cierto nivel de madurez cuando implementan los procesos 
claves de dicho nivel, así como los de los niveles anteriores (F. Niessink \& Van Vlient, 1999). Aunque las organizaciones comparan sus funciones de SI/TI (Gordon, 1994) por múltiples razones, las más comúnmente citadas incluyen la justificación de las inversiones, evaluación del desempeño y la mejora de las funciones de los departamentos de TI (Gordon, 1994).

Planeación y Control. Nolan (1973) menciona que las actividades de planeación, organización y control asociadas a la administración de TI cambian con respecto al tiempo, y que evolucionarán en patrones aproximadamente relacionados a las etapas de madurez. Además, el cumplimiento de una meta específica en un área significa una planeación y control mejorada en la implementación de procesos asociados con dicha área; en consecuencia, indica si es probable que esos procesos sean efectivos, repetibles y durables (C.M.U., 2000). Adicionalmente, la Tabla 1 muestra la intersección de dichas teorías mediante asteriscos, con lo cual se construyó el instrumento aplicado. Además, la Figura 1 muestra en forma gráfica la intersección de dichas teorías.

Tabla 1. Teorías Base

\begin{tabular}{|c|c|c|c|c|}
\hline $\begin{array}{l}\text { Teorías Base } \\
\text { Áreas comunes }\end{array}$ & $\begin{array}{l}\text { Teoría } \\
\text { de } \\
\text { Etapas }\end{array}$ & CMMI & $\begin{array}{c}\text { IT } \\
\text { Service } \\
\text { CMM }\end{array}$ & Benchmarking \\
\hline $\begin{array}{l}\text { 1. Satisfacción del } \\
\text { Usuario }\end{array}$ & * & * & $*$ & * \\
\hline 2. Personal & * & * & & * \\
\hline $\begin{array}{ll}\text { 3. } & \text { Recursos } \\
& \text { Financieros } \\
\end{array}$ & * & * & $*$ & * \\
\hline 4. Tecnología & * & * & * & * \\
\hline $\begin{array}{ll}\text { 5. } & \begin{array}{l}\text { Alineamiento } \\
\text { hacia el Negocio }\end{array} \\
\end{array}$ & * & * & & * \\
\hline 6. $\begin{array}{l}\text { Portafolio de } \\
\text { Aplicaciones }\end{array}$ & * & * & & * \\
\hline 7. La Organización & * & * & * & * \\
\hline $\begin{array}{l}\text { 8. Planeación y } \\
\text { Control }\end{array}$ & * & * & & \\
\hline
\end{tabular}




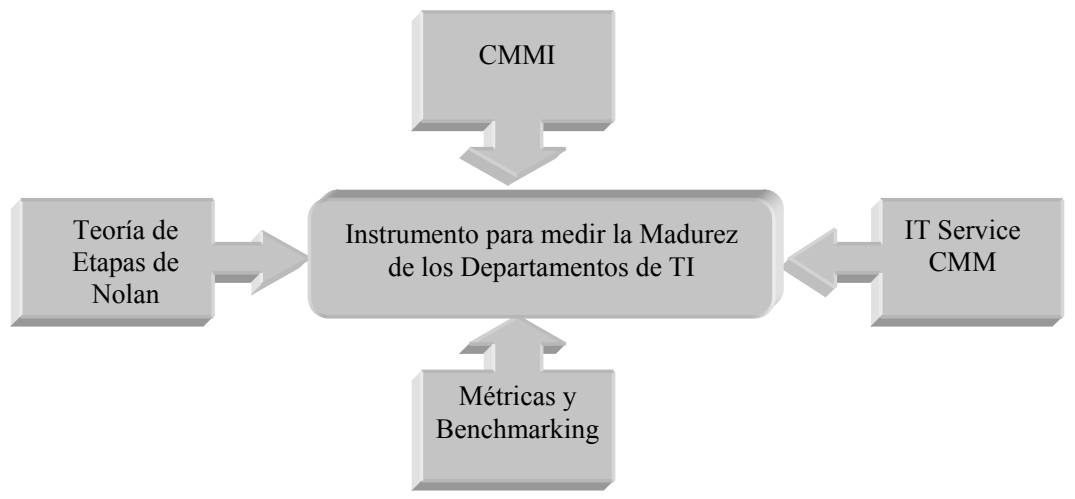

Figura 1. Construcción del instrumento de medición

\section{METODOLOGÍA DE INVESTIGACIÓN}

La presente investigación pretende identificar aspectos relevantes de las cuatro teorías que aporten contenido en la creación del instrumento. Se utilizaron un total de 32 variables en la construcción del instrumento. Cada pregunta utilizó una escala de 7 de Liekert. A continuación se listan las variables utilizadas junto con su definición operacional.

1. Relación con el departamento de TI: forma en que interactúan los usuarios operativos con los administradores de las TI.

2. Tiempo de Respuesta: tiempo que tarda el departamento de TI en entregar el estado de un requerimiento levantado por un usuario operativo.

3. Capacitación: El número de eventos a los que asiste un usuario tanto administrador como operario de los sistemas de información.

4. Entendimiento: grado de acercamiento entre lo que pide el usuario operativo y lo que proporciona el departamento de TI.

5. Participación: grado de acercamiento que tienen los usuarios operativos en relación a los departamentos de TI.

6. Actitud del departamento de TI: tipo de respuesta que el usuario operativo recibe al realizar un requerimiento al departamento de TI.

7. Confiabilidad de la Información: grado en que los usuarios de los sistemas de información perciben el manejo y procesamiento de información.

8. Relevancia de la Información: grado de importancia que la organización le da a la información que es almacenada y procesada.

9. Exactitud de la Información: grado de concordancia con que se realizan los cálculos matemáticos y lógicos.

10. Precisión de la información: grado de variación en los resultados arrojados por los sistemas de información.

11. Comunicación con el departamento de TI: calidad del vínculo que existe entre los usuarios operativos con los usuarios del departamento de TI. 
12. Tiempo de respuesta a nuevos requerimientos: tiempo que tarda el departamento de TI en atender las solicitudes a nuevos requerimientos.

13. Alcance de la Información: impacto que tiene la información sobre las decisiones que toma la organización.

14. Personal Involucrado: perfil de los integrantes del departamento de TI.

15. Grado de Especialización: grado de preparación específica que tienen los integrantes del departamento de TI.

16. Presupuesto: nivel de inversión/gasto que ejerce el departamento de TI.

17. Comunicaciones: alcance que tienen las telecomunicaciones dentro de la organización.

18. Pronóstico: forma para poder determinar necesidades en el futuro.

19. Investigación: grado en que son establecidos proyectos de investigación.

20. Rentabilidad: grado en que las TI aprovechan los recursos utilizados por la organización.

21. Valor Agregado: grado que aportan las TI al valor de la organización.

22. Reconocimiento: importancia que da la organización al departamento de TI.

23. Toma de Decisiones: grado en que las TI ayudan en la toma de decisiones.

24. TPS: grado de uso de los sistemas transaccionales.

25. MIS: grado de uso de los sistemas de información administrativos.

26. MSS: grado de uso de los sistemas de información de soporte.

27. EIS: grado de uso de los sistemas de información para ejecutivos.

28. Institucionalización: grado en que son establecidos los estándares y evidencias reales en los procesos del departamento de TI.

29. Desempeño: nivel de los mecanismos que aseguran el correcto desempeño del departamento de TI.

30. Planeación: grado en que son establecidos y llevados a cabo los lineamientos para la planeación estratégica por parte del departamento de TI.

31. Control: nivel de los mecanismos que aseguran tener bajo control las TI dentro de la organización.

32. Mejora Continua: grado en que está establecido un plan que manifiesta el control en la calidad de los procesos.

\section{Población Objetivo y Unidad de Análisis de Datos}

Los usuarios de TI en las organizaciones son los que más conocen acerca de la situación real que tiene el departamento de TI. Es por eso que se aplicó el instrumento a dichos usuarios, por lo cual son identificados como la unidad de análisis para esta investigación. Entendiéndose como usuarios tanto personas que interactúan con las TI de manera operativa como personas que las administran. 


\section{Instrumento de medición}

Tomando como referencia de desarrollo las teorías base anteriormente citadas y después de una revisión literaria se encontraron los puntos en común, dando como resultado la construcción de un cuestionario, el cual es el instrumento inicial utilizado en la investigación. Posteriormente, se realizó un estudio piloto con 10 personas con el fin de identificar cualquier problema con la redacción del instrumento. Asimismo, se utilizó estadística descriptiva con el fin de verificar si los resultados obtenidos tuvieran un comportamiento normal de forma tal que se eliminara la posibilidad de respuestas sesgadas. Una vez resueltos los problemas de redacción presentes en el cuestionario y medir su confiabilidad, se obtuvo el instrumento aplicado.

\section{Recolección de información}

Para esta investigación se encuestaron empleados de 60 empresas. La participación se limitó a empresas catalogadas como pequeñas y medianas de acuerdo a SEDEC ${ }^{1}$, debido a que las empresas grandes en donde se aplicó la encuesta declinaron participar en el estudio. Se recolectaron los datos utilizando preguntas con respuestas en una escala de Likert de 7 puntos. Literatura previa (Hair, Anderson, Tatham, \& Black, 1998; Stapleton, 1997) recomienda que la relación entre el número de observaciones y variables no sea menor a cinco con el fin de encontrar valores significativos. Además, siguiendo el proceso definido por Lethen (1996) se calculó 126 encuestas como el número mínimo necesario. Con el fin de cuidar este aspecto se intentaron aplicar 200 encuestas, de las cuales se obtuvieron 126 encuestas utilizables.

Para la aplicación de la encuesta se solicitó a las organizaciones la forma preferida de respuesta, ya sea personalmente o por correo electrónico. De las encuestas utilizables aproximadamente, el $80 \%$ se aplicaron mediante interacción cara-a-cara, y el resto se recibieron por correo electrónico.

Algunas encuestas recibidas se desecharon debido a que estaban incompletas o algunas preguntas tenían una doble respuesta y otras organizaciones nunca respondieron la encuesta recibida. Las industrias encuestadas para el presente estudio y su porcentaje de participación en el mismo se muestran en la Tabla 2.

Tabla 2. Industrias que participaron en el estudio

\begin{tabular}{|r|r|r|r|r|r|r|}
\hline Gobierno & Construcción & Educación & Salud & Servicios & $\begin{array}{c}\text { Telecomuni- } \\
\text { caciones }\end{array}$ & $\begin{array}{c}\text { Agrope- } \\
\text { cuaria }\end{array}$ \\
\hline 10 & 20 & 5 & 25 & 31 & 10 & 25 \\
\hline $7.9 \%$ & $15.9 \%$ & $4.0 \%$ & $19.8 \%$ & $24.6 \%$ & $7.9 \%$ & $19.8 \%$ \\
\hline
\end{tabular}

${ }^{1}$ Secretaría de Desarrollo Económico del Estado de Aguascalientes, México 


\section{DISCUSIÓN DE RESULTADOS}

El análisis de factores exploratorio permitió descubrir las dimensiones del conjunto de variables medidas en el estudio, el cual dio como resultado los factores críticos que permitieron medir el nivel de madurez de los departamentos de TI de las organizaciones encuestadas. Para efectos de análisis, se utilizó la sugerencia de Kline (2002) de usar como valor mínimo de carga 0.60. En nuestro caso, se consideró adecuado utilizar al menos 0.65 como valor mínimo de carga. Dicho análisis identificó un total de nueve factores. Es importante mencionar que se encontraron seis factores útiles, los cuales están compuestos por al menos un mínimo de 3 variables para poder ser evaluados y considerados, y tres no cumplen con esta característica, por lo que fueron eliminados.

Una vez obtenidos los resultados rotados del análisis de factores para las 62 preguntas utilizadas en el instrumento de medición, se obtuvo la Tabla 3 , la cual muestra cada uno de los factores con sus variables asociadas. También se presenta el valor de carga de cada una de las variables.

Tabla 3. Factores Resultantes

\begin{tabular}{|c|c|c|}
\hline Factor & Variables & Carga \\
\hline \multirow{5}{*}{$\begin{array}{l}\text { Satis- } \\
\text { facción del } \\
\text { Usuario }\end{array}$} & 24. Confiabilidad de la información que proporcionan las TI & .6970 \\
\hline & 25. Actitud que tiene el departamento de TI & .7410 \\
\hline & 54. Relación con el personal del departamento TI & .7287 \\
\hline & $\begin{array}{l}\text { 55. Nivel de exactitud que ofrece la información proporcionada por } \\
\text { los sistemas de información (cálculos) }\end{array}$ & 60002 \\
\hline & $\begin{array}{l}\text { 57. Precisión de la información que proporcionan los sistemas de } \\
\text { información }\end{array}$ & .6602 \\
\hline \multirow{6}{*}{$\begin{array}{l}\text { Portafolio } \\
\text { de Aplica- } \\
\text { ciones }\end{array}$} & $\begin{array}{l}\text { 37. Grado de avance en la operación y desarrollo de los sistemas } \\
\text { ejecutivos (EIS) en la organización }\end{array}$ & .7548 \\
\hline & $\begin{array}{l}\text { 43. Grado de avance en la operación y desarrollo de los sistemas } \\
\text { gerenciales (MIS) en la organización }\end{array}$ & .7772 \\
\hline & 44. Grado de aceptación de los sistemas MIS en la organización & .7821 \\
\hline & $\begin{array}{l}\text { 45. Grado de avance en la operación y desarrollo de los MSS en la } \\
\text { organización (EIS,ES,DSS) }\end{array}$ & .8598 \\
\hline & 48 Grado de aceptación MSS en la organización & .7807 \\
\hline & 49. Grado de aceptación de los EIS en la organización & .7988 \\
\hline \multirow{3}{*}{$\begin{array}{l}\text { Capital } \\
\text { Humano }\end{array}$} & 11. Nivel de profesionalización dentro del departamento de TI & 6775 \\
\hline & 21. Número de técnicos en el departamento de TI & .7969 \\
\hline & 30. Número de personas con postgrado en el departamento de TI & .7926 \\
\hline \multirow{5}{*}{$\begin{array}{l}\text { Estructura } \\
\text { Organiza- } \\
\text { cional }\end{array}$} & $\begin{array}{l}\text { 3. Tiempo de respuesta que emplea el departamento de TI para el } \\
\text { desarrollo de un nuevo requerimiento }\end{array}$ & 6453 \\
\hline & $\begin{array}{l}\text { 5. Grado de existencia de mecanismos que permitan el manejo } \\
\text { adecuado de las contingencias en la organización }\end{array}$ & .7430 \\
\hline & $\begin{array}{l}\text { 6. Grado en que se encuentran definidos los mecanismos que } \\
\text { permiten un seguimiento de los servicios de TI proporcionados a la } \\
\text { organización }\end{array}$ & .7158 \\
\hline & $\begin{array}{l}\text { 7. Avance en el desarrollo/implantación de mecanismos que } \\
\text { propicien el uso de estándares dentro del departamento de TI }\end{array}$ & .6601 \\
\hline & $\begin{array}{l}\text { 9. Nivel en que se encuentran los mecanismos de evaluación del } \\
\text { desempeño del departamento de TI en la organización }\end{array}$ & 6873 \\
\hline
\end{tabular}




\begin{tabular}{|c|c|c|}
\hline & $\begin{array}{l}\text { 12. Grado en que se considera que la organización promueve y } \\
\text { realiza labores de planeación estratégica sobre TI }\end{array}$ & .6434 \\
\hline & $\begin{array}{l}\text { 15. Grado de avance en la definición de una estructura } \\
\text { organizacional para el departamento de TI }\end{array}$ & .6085 \\
\hline & $\begin{array}{l}\text { 16. Grado de importancia en que se encuentran definidos los } \\
\text { mecanismos que aseguren la entrega en tiempo y forma de los } \\
\text { servicios de TI proporcionados a la organización }\end{array}$ & .6159 \\
\hline \multirow{6}{*}{$\begin{array}{l}\text { Modelo de } \\
\text { Calidad }\end{array}$} & $\begin{array}{l}\text { 50. Grado de implementación de los planes de mantenimiento de las } \\
\text { TI }\end{array}$ & .6069 \\
\hline & $\begin{array}{l}\text { 51. Grado de avance en la documentación de los procesos del } \\
\text { departamento de TI }\end{array}$ & .6901 \\
\hline & $\begin{array}{l}\text { 58. Grado alcanzado de los instrumentos de mejora continua } \\
\text { implementados por el departamento de TI }\end{array}$ & .7175 \\
\hline & $\begin{array}{l}\text { 59. Medida en que existen mecanismos que aseguren el correcto } \\
\text { uso del presupuesto de TI en la adquisición de tecnología }\end{array}$ & .7702 \\
\hline & $\begin{array}{l}\text { 60. Grado de avance en el uso de herramientas para administración } \\
\text { de proyectos en el departamento de TI }\end{array}$ & .7282 \\
\hline & $\begin{array}{l}\text { 62. Grado en que son implementados en la organización los } \\
\text { estándares y métricas de calidad }\end{array}$ & .6713 \\
\hline \multirow{5}{*}{$\begin{array}{l}\text { Recursos } \\
\text { Finan- } \\
\text { cieros }\end{array}$} & 14. Nivel de presupuesto del departamento de TI & .6546 \\
\hline & 26. Nivel de presupuesto asignado al hardware & .7395 \\
\hline & $\begin{array}{l}\text { 27. Grado de infraestructura de telecomunicaciones de la } \\
\text { organización }\end{array}$ & .6343 \\
\hline & 33. Nivel de presupuesto asignado al software & .6677 \\
\hline & $\begin{array}{l}\text { 38. Nivel de presupuesto asignado a telecomunicaciones (redes, } \\
\text { cableado, enlaces, etc.) }\end{array}$ & .8073 \\
\hline F7 & $\begin{array}{l}\text { 28. Grado de independencia del departamento de TI en relación con } \\
\text { la organización }\end{array}$ & .7380 \\
\hline F8 & $\begin{array}{l}\text { 17. Nivel de re-uso de métodos, técnicas, herramientas, planes, } \\
\text { documentación, diseño, código, etc., sobre TI }\end{array}$ & .7641 \\
\hline F9 & $\begin{array}{l}\text { 31. Apreciación personal del nivel de comprensión de los sistemas } \\
\text { de información }\end{array}$ & .6092 \\
\hline
\end{tabular}

A continuación se describe la forma en que fueron nombrados cada uno de los factores identificados. Algunos de ellos coinciden exactamente con los previamente identificados en las teorías base. A otros, se les debió crear un nuevo nombre debido a las variables que se agrupan en ellos.

Satisfacción del Usuario agrupa el conjunto de variables que evalúan la apreciación que tienen los usuarios operativos hacia los usuarios administrativos de los departamentos de TI de las organizaciones y viceversa. Es decir, se identifican el conjunto de variables que se asocian al cumplimiento de las expectativas de los usuarios.

Portafolio de Aplicaciones presenta la evaluación de los diferentes tipos de sistemas con los que cuentan las organizaciones. Las variables asociadas a él miden el impacto que tiene para los usuarios cada uno de los tipos de sistemas que cualquier organización pudiera usar en sus operaciones.

Capital Humano evalúa el personal con el que cuentan las empresas para la operación y administración de los recursos computacionales. Se agruparon las variables que miden el número nivel que tienen los recursos 
humanos que laboran en el departamento de TI, lo cual podría impactar en el funcionamiento, forma, y nivel de eficiencia del departamento.

Estructura Organizacional presenta la evaluación de aspectos relacionados con la gestión de TI. En él se observa que están involucrados aspectos correspondientes a la estructura organizacional de los departamentos de TI de las organizaciones tales como tiempos de respuesta, forma en que se realizan las contingencias para mitigar los riesgos, cómo se realizan los seguimientos de los diferentes servicios prestados, implementaciones de estándares, indicadores de desempeño y planeación estratégica. Todos estos aspectos confirman la estructura organizacional por la que se desenvuelven las tecnologías de información.

Modelo de Calidad describe los procesos que aseguran la calidad de los productos y servicios que el departamento de TI brinda a la organización. Las variables medidas intentan medir la importancia que tienen los procesos que aseguren la calidad de los productos y servicios que el departamento de TI brinda a la organización, lo cual es relevante para llegar a tener procesos eficientes y efectivos. En consecuencia, impactan el nivel de madurez de los departamentos de TI.

Al agrupar las variables relacionadas con la inversión y la administración de los recursos financieros se obtiene el factor Recursos Financieros. Es importante saber cuál es el nivel de presupuesto que se destina a los departamentos de TI. Si un departamento no tiene presupuesto suficiente, será imposible cumplir con todas las tareas imprescindibles así como mejorar los recursos existentes de TI. Mucho menos será posible el incorporar TI emergentes que puedan coadyuvar al fortalecimiento de las operaciones de la organización.

Los factores 7, 8 y 9 sólo agrupan una variable, respectivamente, lo cual indica que en realidad no podrían ser factores. Entonces, se considera que las posibles causas que afectaron en las respuestas de las preguntas asociadas pudieron ser debido a que los encuestados pudieron haber contestado al azar sin entender o pedir la clarificación de las preguntas, o que existe un concepto usado de forma diferente dentro de sus organizaciones en los conceptos asociados, o el hecho de que desconocen si del departamento TI aplica dicho concepto.

Teniendo integradas las respuestas de los 126 cuestionarios aplicados se procedió a determinar la confiabilidad de cada uno de los factores propuestos, ya que es necesario establecer la consistencia interna de los reactivos con respecto a la escala de medida. Es decir, obtener el nivel de confiabilidad con la prueba de Alpha de Cronbach. Kerlinger (2000) sugiere que un factor es relevante cuando el valor de Alpha de Cronbach es al menos de .70, lo cual representa la regla utilizada en el presente estudio. La Tabla 4 presenta los resultados de confiabilidad del análisis de factores, y posteriormente se presenta el análisis de los valores obtenidos. 
Tabla 4. Confiabilidad de los Factores

\begin{tabular}{rrrrrrr}
\hline Factor & $\mathbf{1}$ & $\mathbf{2}$ & $\mathbf{3}$ & $\mathbf{4}$ & $\mathbf{5}$ & \multicolumn{1}{c}{$\mathbf{6}$} \\
\hline No. de elementos & \multicolumn{1}{c}{5} & \multicolumn{1}{c}{6} & \multicolumn{1}{c}{3} & \multicolumn{1}{c}{8} & \multicolumn{1}{c}{6} & \multicolumn{1}{c}{4} \\
Media & 26.76 & 24.14 & 8.47 & 35.59 & 25.97 & 17.44 \\
Varianza & 35.05 & 84.55 & 16.97 & 110.36 & 75.37 & 30.58 \\
Desviación estándar & 5.92 & 9.195 & 4.12 & 10.505 & 8.681 & 5.529 \\
Factor de asimetría & -0.9068 & -0.4383 & 1.116 & -0.4081 & -0.6075 & -0.3786 \\
Curtosis & 0.7416 & -0.7126 & 0.6305 & -0.6498 & -0.4198 & -0.3968 \\
Alfa estandarizada & 0.8692 & 0.9278 & 0.7217 & 0.933 & 0.9347 & 0.8831 \\
Correlación promedio & 0.574 & 0.6883 & 0.4661 & 0.6408 & 0.7064 & 0.6564 \\
de elementos & & & & & \\
Alfa de Cronbach & 0.8673 & 0.9278 & 0.7202 & 0.9328 & 0.934 & 0.8828 \\
\hline
\end{tabular}

La principal intención de esta investigación es el identificar los factores que intentan medir el nivel de madurez de los Departamentos de TI de las Organizaciones del Estado de Aguascalientes por medio de la aplicación de una herramienta de diagnóstico. Además, identificar cuáles son considerados como relevantes. Se pudo identificar que existen un total de seis factores, todos ellos considerados como relevantes.

En el estudio se obtuvo un valor estadísticamente significativo, pero durante la aplicación de las encuestas cara-a-cara se pudieron capturar - en muchos de los casos - una serie de opiniones adicionales que de forma libre los encuestados vertieron acerca de las preguntas de la encuesta. Estas opiniones nos dieron una idea de la relevancia práctica que tienen los resultados obtenidos. Tanto los valores numéricos de confiabilidad así como las opiniones capturadas se describen a continuación para cada factor identificado.

La confiabilidad más alta reportada correspondió al factor Modelo de Calidad, con un coeficiente de 0.9340 , por lo cual se acepta dicho factor como relevante. Pero al analizar las opiniones recibidas observamos que aunque esto es un valor significativo estadísticamente, no indica que en la práctica las organizaciones en estos momentos lleven a cabo documentación de sus procesos o implementación de estándares y métricas de calidad. Lo verdaderamente importante es que están realizando acciones encaminadas a lograr lo anterior, y en el peor de los casos, se encuentran conscientes de la gran importancia que tiene esto en la correcta operación y administración de los servicios de TI, lo cual presenta un panorama bastante alentador en cuanto a la profesionalización de las TI en las organizaciones.

A continuación se identificó la importancia que las organizaciones le reconocen a la Estructura Organizacional, ya que el valor de confiabilidad reportado es de 0.9328 . Con respecto a la relevancia práctica de este factor, observamos que esto indica que las organizaciones están conscientes de que sin un adecuado seguimiento a los servicios/proyectos de TI no puede asegurarse la terminación a tiempo y dentro de presupuesto 
$\mathrm{y}$, en consecuencia, la correcta operación de los mismos. Además, llama sobremanera la atención el hecho de que consideren importante el tema del aseguramiento de riesgos, ya que en la práctica son pocas las que, de una manera ordenada y basada en alguna metodología, cuentan con un plan de contingencia y generan medidas tendientes a mitigar dichos riesgos. El hecho de que el tema de gobierno corporativo se encuentra en boga en las diferentes organizaciones, no sólo en el estado, sino a nivel global, está incidiendo fuertemente en la conciencia de las organizaciones sobre la importancia de las TI, así como de su estructura organizacional.

Posteriormente, el tercer valor de confiabilidad más alto corresponde al Portafolio de Aplicaciones (0.9278). En cuanto a su relevancia práctica, observamos que es importante el hecho de que las organizaciones reconozcan en el portafolio de aplicaciones el motor del trabajo diario de cada una de las actividades que se desempeñan dentro de la organización. De esta forma, se podrán concentrar en un proceso de mejora continua de los SI. Esto podría indicar el hecho de que las organizaciones están conscientes de los diferentes tipos de Sistemas de Información (SI) a implementar dentro de un todo en la infraestructura de TI. Pero en la práctica podemos comprobar que la gran mayoría de ellas se encuentra en la generación de elementales sistemas ejecutivos, en los mejores casos en la adopción de plataformas integrales, o saliendo de una dura implementación de sus sistemas transaccionales, dejando para después el tema de los sistemas de soporte en la toma de decisiones. En resumen, las organizaciones no le han prestado la importancia debida al desarrollo de sistemas que ayuden a los mandos intermedios y gerenciales, concentrando sus esfuerzos en sistemas transaccionales.

En cuarto lugar, con un valor de 0.8828 se encuentran los Recursos Financieros. Además, se observó que las organizaciones reconocen que una correcta aplicación de los recursos, tanto en infraestructura como en servicios de TI, corresponde al éxito de los proyectos. En la práctica suena contradictorio el hecho de que los presupuestos no existan como tal, y que la gente del Departamento de TI tenga que negociar la adquisición de infraestructura o la generación de nuevos proyectos. Lo que se vuelve una obligación en estos escenarios es la justificación y la sustentabilidad de dichos proyectos por parte del departamento de TI. En la medida que sustente con beneficios palpables para la organización el gasto en TI, éste puede convertirse a la vista de la misma en una inversión - y no un gasto que reditué ya no en ventajas competitivas sino en dividendos para ésta y para el mismo Departamento de TI en la asignación y operación de dichos presupuestos.

En cuanto a la Satisfacción del Usuario, su nivel de confiabilidad obtenido es de 0.8673. Adicionalmente, al analizar su relevancia práctica observamos que esto puede indicar que operativamente, en el ánimo del logro de metas en la organización, la persona queda relegada por debajo de 
otros factores. Por otro lado, en la práctica, si el usuario no está siendo atendido de manera correcta o no se le ha tomado en cuenta para la implantación/adopción de tecnología, puede convertirse en un riesgo no sólo para el proyecto sino para la organización misma, pues la gran mayoría de los procesos de las organizaciones no cuenta con un grado de tecnificación elevado, por lo que es necesario el capital humano para su consecución. Además, es reconocido que el capital humano es un elemento de lo más importante para que una empresa logre el éxito primero, y que lo sostenga posteriormente (Drucker, 1992, 1993, 1999).

El factor Capital Humano reporta el coeficiente más bajo de todos los factores evaluados (.7202). Analizando la relevancia práctica, se detectó una contradicción aparente, ya que las organizaciones reconocen la importancia de contar con el personal adecuado para la correcta administración de los recursos. Adicionalmente, las organizaciones buscan que una tarea determinada dentro de la empresa sea desempeñada por aquella persona que cuenta con el mejor perfil para realizarla. Por el contrario, en la práctica las organizaciones no han encontrado la manera de identificar adecuadamente la capacidad del capital humano, generalmente se le da preferencia al factor técnico y en consecuencia, el departamento de TI se queda únicamente en el nivel técnico aunque se cuenta dentro de la plantilla con personal con estudios de postgrado.

\section{CONCLUSIONES Y RESULTADOS OBTENIDOS}

$\mathrm{Al}$ analizar los resultados se pudo conocer una situación interesante, la cual se podría decir que fue la historia vista desde dos puntos de vista diferente. Por un lado, al analizar los datos capturados en las encuestas se pudo observar cuáles son los factores que son considerados por los usuarios de TI como críticos para que se pueda dar un soporte eficiente a la organización y se puedan cumplir sus metas. Esto no puede ser ignorado, ya que las TI son una herramienta más que puede ayudar en los planes estratégicos. El tener o no las TI adecuadas podría significar la diferencia entre el éxito y el fracaso en muchas de las organizaciones encuestadas.

El factor Modelo de Calidad tuvo la confiabilidad más alta (.9340), por lo que los encuestados identifican como de mayor importancia el que los trabajos se lleven a cabo bien y a la primera. Es decir, consideran que el trabajo se debe llevar a cabo con la mejor calidad posible, ya que de esta manera se estarán teniendo procesos robustos que proporcionen soporte a los usuarios. De esta manera, los usuarios pueden desempeñar sus actividades de la mejor manera posible. Impactando todo esto en la calidad y la productividad global de la empresa.

El factor Estructura Organizacional fue identificado como segundo en importancia (.9328). Esto implica que para que los procesos relacionados con las TI sean maduros es muy importante que las organizaciones 
cuenten con una estructura sólida y bien establecida. El hecho de que cada usuario conozca qué actividades y responsabilidades tiene dentro de la organización permite definir claramente las actividades, así como deslindar responsabilidades en casos que así lo requieran. Entonces, los empleados de las TI estarán conscientes de la importancia de que las actividades se realicen a tiempo y dentro de presupuesto, pero siempre dentro de los estándares de calidad.

Los participantes identificaron al Portafolio de Aplicaciones (.9278) como muy relevante. Esto implica que las organizaciones requieren una variedad suficiente de aplicaciones de TI y SI para poder realizar sus actividades de forma óptima de forma tal que les permita permanecer competitivos. En un mundo globalizado es imprescindible que las organizaciones que marcan la pauta utilicen y apliquen la tecnología en sus procesos. Entonces, se hace absolutamente necesario que los procesos para la aplicación/generación de tecnología deben ser eficientes y con calidad.

A continuación, en orden numérico tenemos el factor Recursos Financieros (.8828). La tecnología para poder ser desarrollada/implantada/mantenida demanda inversiones. Asimismo, para que los procesos relacionados con ello sean eficientes es necesario contar con personal calificado. En consecuencia, si no existen recursos financieros no podrá contarse con tecnología adecuada administrada y utilizada por personal calificado. Ahora bien, esto no quiere decir que la inversión pura generará TI adecuadas. Esto se logrará mediante la convergencia de todos los elementos existentes en la organización.

El factor Satisfacción del Usuario (.8673) sigue en el orden. Este factor fue identificado como muy relevante, ya que la peor inversión en tecnología es aquella en la que la TI no es utilizada. Es decir, no es suficiente tener la mejor TI, procesos de calidad y personal calificado, ya que si la tecnología no es utilizada, en consecuencia, no se puede obtener provecho de la misma, ni optimizar recursos ni incrementar la productividad. Todo esto, en detrimento de la organización, lo que quizá podría desencadenar la desaparición de la empresa.

Sorprendentemente, en último lugar de confiabilidad tenemos al Capital Humano (.7202). Es bien sabido que la mejor tecnología no puede funcionar, ni la empresa pude existir como tal si no se tiene capital humano capacitado y eficiente. Este resultado pudo deberse a que los respondientes se concentraron más en la tecnología misma que en quienes la desarrollan o la utilizan para darle valor a los procesos del negocio. No quiere decir que no sea relevante, simplemente pudo ser que la consideraron como "de facto", es decir, las personas vienen junto con la tecnología.

Pero, por otro lado, los comentarios que se recibieron durante la aplicación de las encuestas hablan de una historia diferente, ya que si bien es cierto 
que los factores detectados son críticos y muy importantes, en la realidad pudimos observar que no necesariamente son llevados a la práctica. Es decir, son considerados como de extrema importancia pero no quiere decir que se les presta la atención requerida. Lo que sí podemos afirmar es que las organizaciones participantes quedaron alertadas de esta situación, lo cual podría impactar en su planeación futura.

Ahora bien, al finalizar el presente trabajo y darles a conocer los resultados pudimos observar que en muchos de los casos, cambiaron su forma de interactuar con los directivos, ya que los gerentes de TI tenían en sus manos elementos probatorios de que la TI es muy relevante pero que necesita más atención para que la empresa transforme el presupuesto de TI de ser un mero gasto a una total inversión.

\section{RECOMENDACIONES}

Basándose en los resultados obtenidos creemos importante recomendar que es fundamental incluir al personal al momento de realizar la planeación estratégica de las TI en las organizaciones, ya que en muchas de las ocasiones, los empleados son los que realmente saben lo que se necesita para la operación diaria. Si esto lo aunamos a las necesidades que los altos mandos tienen de TI, en consecuencia, se puede llevar a cabo una planeación más rica de los recursos. Esto podría resultar en una aplicación de las TI que permita a las organizaciones ya sea, adquirir o sostener ventajas competitivas.

Por otro lado, es importante que las organizaciones midan con relativa frecuencia el estado que guardan sus departamentos de TI, ya que esto les permitirá medir el estado actual y compararlo con lo planeado. De esta forma, podrían detectar y/o prevenir desviaciones a tiempo, de forma tal, que puedan tomar las medidas adecuadas y así coadyuvar a la organización a lograr las metas establecidas en los planes estratégicos. Esto podría demostrar que las TI no son un gasto más, sino que son una inversión altamente redituable para la organización.

\section{LIMITACIONES}

Es importante mencionar que la invitación se realizó a todas las empresas consideradas dentro del padrón de la Secretaría de Desarrollo Económico del Estado de Aguascalientes, México; y sabiendo que las que aceptaron participar pudiera haber originado que la muestra podría no ser representativa de las organizaciones Mexicanas, siendo esta la principal debilidad del estudio realizado; éste hecho podría haber influido en los resultados. Los resultados finales podrían ser diferentes si se reproduce el presente estudio con una muestra de tamaño más grande totalmente representativa y seleccionada aleatoriamente. Además, en algunos casos no se tuvo el control de quien respondió la encuesta ya que se respondió por correo electrónico, esto podría haber provocado que no entendieran algunas preguntas, y en consecuencia, respondido inadecuadamente; o que 
no se leyera detenidamente y se respondiera de forma aleatoria. Pudieran existir algunos otros factores que no se hubieran considerado o identificado durante la ejecución del presente estudio que podrían haber afectado los resultados obtenidos. Finalmente, aunque los resultados obtenidos son alentadores y presentan validez interna es importante mencionar que el instrumento carece de una fuerte validez externa.

\section{TRABAJOS FUTUROS}

Se espera aplicar el instrumento con una cobertura regional a una muestra representativa de las organizaciones Mexicanas con el fin de fortalecer aún más el instrumento utilizado. Esto con el fin de, posteriormente, aplicarlo de forma nacional y poder confirmar - o rectificar - los resultados obtenidos.

\section{BIBLIOGRAFÍA}

Agrawal, M., \& Chari, K. (2007). Software Effort, Quality, and Cycle Time: A Study of CMM Level 5 Projects. IEEE Transactions on Software Engineering, 33(3), 145-156.

Brynjolfsson, E., \& Hitt, L. (1996). Paradox Lost? Firm-level evidence on the returns to information systems spending. Management Science, 42(4), 541-558.

C.M.U., C. M. U. (2000). Capability Maturity Model ${ }^{\circledR}$ Integration (CMMISM), Version 1.1 (No. CMU/SEI-2002-TR-028

ESC-TR-2002-028). Pittsburgh, PA: Software Engineering Institute, Carnegie Mellon University.

Crook, C. W. (1997). A study of the career orientations of information systems students and professionals. Paper presented at the Special Interest Group on Computer Personnel Research Annual Conference, San Francisco, CA, USA.

Delmonte, A. J. (2003). Information Technology and the Competitive Strategy of Firms. Journal of Applied Management and Entrepreneurship, 8(1), 115-129.

Drucker, P. F. (1992). Managing for the Future: The 1990s and Beyond. New York, NY: Truman Talley Books/Dutton.

Drucker, P. F. (1993). The Effective Executive. Harper Business Press, New York.

Drucker, P. F. (1999). Management Challenges for the 21st Century. Harper Business Press, New York.

Due, R. T. (1996). The New Productivity Metrics. Information Systems Management, 13(4), 60-62.

Farris, J., Letens, G., Van Aken, E. M., Ellis, K., \& Boyland, J. (2007). A Structured Approach for assessing the Effectiveness of Engineering Design Tools in New Product Development. Engineering Management Journal, 19(2), 31-39.

Ginzberg, M. J., \& Baroudi, J. J. (1992). Career Orientations of I.S. Personnel. Computer Personnel, 14(2), 15-29. 
Gordon, S. (1994). Benchmarking The Information Systems Function. Retrieved 5/01/2005, 2005, from http://faculty.babson.edu/gordon/papers/F94BENCH.HTM

Hair, J., Anderson, R., Tatham, R., \& Black, W. (1998). Multivariate Data Analysis (5th Edition). Upper Sadlle River, NJ: Prentice-Hall.

Hitt, L., \& Brynjolfsson, E. (1996). Productivity, business profitability, and consumer surplus: Three different measures of Information Technology value. MIS Quarterly, 20(2), 121-142.

Janssen, M., Gortmaker, J., \& Wagenaar, R. W. (2006). Web Service Orchestration in Public Administration: Challenges, Roles, and Growth Stages. Information Systems Management, 23(2), 44-.

Kerlinger, F. N., \& Lee, H. B. (2000). Foundations of Behavioral Research. Fourth Edition. New York: Harcourt College Publishers.

King, J. L., \& Kraemer, K. L. (1984). Evolution and Organizational Information Systems: An Assessment of Nolan's Stage Model. Communications of the ACM, 27(5), 1-10.

Kline, P. (2002). An easy Guide to Factor Analysis. New York, NY: Routledge.

Leem, C. S., \& Kim, I. (2004). An integrated evaluation system based on the continuous improvement model of IS performance. Industrial Management + Data Systems, 104(1/2), 115-128.

Leem, C. S., \& Yoon, Y. (2004). A maturity model and an evaluation system of software customer satisfaction: the case of software companies in Korea. Industrial Management + Data Systems, 104(3/4), 347-.

Lethen, J. (1996). Statistics 30x Class Notes, 1996

Niessink, F., \& Van Vlient, H. (1999). The Vrije Universiteit IT Service Capability Maturity Model (Technical No.

IR-463, Release L2-1.0). Amsterdam: Vrije Univeristeit Amsterdam.

Niessink, F., \& van Vlient, H. (2000). Software Maintenance from a Service Perspective. Journal of Software Maintenance: Research and Practice, 12(2), 103-120.

Niessink, F., \& van Vliet, H. (1999). The Vrije Universiteit IT Service Capability Maturity Model (No. IR-463). Amsterdam, The Netherlands: Vrije Universiteit Amsterdam.

Nolan, R. L. (1973). Managing the Computer Resource: A Stage Hypothesis. Communications of the ACM, 16(17), 399 - 405.

Pressman, R. S. (2005). Software Engineering: A Practitioner's Approach, 6/e. New York, N.Y.: Mc Graw-Hill.

Sommerville, I. (2006). Software Engineering. 8/e. London, U.K.,: Addison-Wesley.

Stapleton, C. D. (1997). Basic concepts and Procedures of Confirmatory Factor Analysis. Annual Meeting of the Southwest Education Research Association. 
van Vlient, H. (1999, 2005-03-31). The IT Service CMM in a Nutshell, 2005, from http://www.itservicecmm.org/nutshell.html

Nota del Editor: Las referencias anteriores contienen direcciones de WWW. (a) Dichas direcciones existían a la fecha de publicación pero no hay garantía alguna de que persistan en el tiempo, (b) su contenido puede cambiar en el tiempo y las siguientes versiones pueden no contener la información a la que se hizo referencia, (c) el autor o autores de dichas paginas (no AIS ni RELCASI) son los únicos responsables de su contenido, y (d) el autor de este articulo (no AIS ni RELCASI) es el único responsable de la exactitud de dichas direcciones.

Juan Manuel Gómez Reynoso es Ingeniero en Sistemas Computacionales y estudió la maestría en Informática y Tecnologías Computacionales y la Especialidad en Redes en la Universidad Autónoma de Aguascalientes (UAA), México. Posteriormente, estudió la maestría en Ciencias en Sistemas de Información y el Doctorado en Ciencias en Sistemas de Información en Claremont Graduate University, USA. Es profesor de tiempo completo de la UAA desde 1990. Actualmente es coordinador de la carrera de Ing. en Sistemas Computacionales y de la Maestría en Redes de Computadoras.

Carlos Alejandro Torres Quiñones cursó sus estudios profesionales de licenciatura como Ingeniero en Sistemas Computacionales, posteriormente realizó la Maestría en Informática y Tecnologías Computacionales, ambos grados fueron alcanzados dentro de la Universidad Autónoma de Aguascalientes, México. Actualmente se desempeña como Ingeniero de Software en la empresa SOFTTEK, líder en soluciones de Tecnología de Información y Procesos de Negocio enfocado a satisfacer necesidades de desarrollo, implantación y soporte.

Javier Santiago Cortes López cursó sus estudios profesionales de licenciatura como Ingeniero en Sistemas Computacionales, posteriormente realizó la Maestría en Informática y Tecnologías Computacionales, ambos grados fueron alcanzados dentro de la Universidad Autónoma de Aguascalientes, México. Actualmente Trabaja para el Gobierno Estatal del estado de Aguascalientes, México así como es consultor de diversas organizaciones privadas. 


\section{Política Editorial}

RELCASI está principalmente dirigida a investigadores de habla hispana en el área de Administración de Sistemas de Información (MIS por sus siglas en Inglés). Los artículos son académicamente rigurosos sin sacrificar la claridad, estilo, simplicidad y contribución práctica que los hace atractivos a profesionales de la disciplina. En consecuencia, la audiencia de esta revista está compuesta no sólo por investigadores de MIS, sino también por profesionales y administradores en el área de tecnologías de información.

Todos los artículos son escritos, revisados y publicados en español; sin embargo, estos contendrán título, resumen y palabras claves en español y en inglés.

RELCASI es una publicación arbitrada que se presenta en formato impreso y en línea. La versión impresa de RELCASI está disponible a pedido (y próximamente bajo subscripción). La versión en línea se provee a través de la Asociación de Sistemas de Información. El proceso de evaluación se realizará con al menos dos examinadores. La identidad del editor asociado y de los examinadores no será del conocimiento del autor, y ni los examinadores ni el editor asociado conocerán la identidad del autor. Una ronda del proceso de evaluación tomará alrededor de 90 días.

La revista incluye principalmente artículos de investigación desarrollados con un marco teórico robusto y que incluyan una adecuada revisión de literatura. Los artículos podrán ser de investigación empírica (cualitativa o cuantitativa), conceptuales, encuestas de corrientes de investigación, o encuestas de la industria de TI en países en desarrollo. Los artículos de investigación empírica, deben proveer una amplia justificación $y$ descripción de la colección de datos, metodología y técnicas analíticas. Estudios de caso, artículos pedagógicos, revisión de libros, y debates y ensayos de opinión serán considerados pero no formarán el grueso de la publicación. Artículos con un alto contenido técnico y bajo contenido gerencial/administrativo no son recomendados y sólo serán aceptados cuando sean altamente relevantes o innovadores. Los artículos deberán tener una extensión no mayor a las 8.000 palabras. 


\section{Editorial Policy}

RELCASI is primarily directed to Spanish speaking researchers in the area of Management Information Systems (MIS). Articles will have academic rigor without sacrificing clarity, style, simplicity, and a practical contribution that will also make them attractive to practitioners. Therefore, its audience includes both academics and practitioners of MIS and IT.

Articles are written, reviewed, and published in Spanish; however, their title, abstract, and keywords will also be published in English.

RELCASI is a double-blind peer-reviewed journal that is both in-print and on-line. The print version is currently provided on-demand and we will soon have a subscription service. The on-line version is available through the Association for Information Systems. The double-blind peer-review process will involve an associate editor and a minimum of two academic peers. We aim to have a round of the review process take no more than 90 days.

The journal will primarily comprise of research articles developed with a robust theoretical framework that include an appropriate literature review. The articles could be qualitative or quantitative, conceptual, research stream surveys, or surveys that relate to IT/MIS in developing countries. Empirical research articles must include a clear, comprehensive, and concise description of the methodology, data collection, and analytical techniques used. Case studies, pedagogical articles, book reviews, debates, and opinion papers will be considered but will not make the bulk of the journal. Articles with a high technical and low managerial content are not encouraged but may be accepted if highly relevant or innovative. Articles may not include more than 8,000 words. 